K.-S. Lee

Nagoya Math. J.

Vol. 119 (1990), 93-106

\title{
WHITE NOISE APPROACH TO GAUSSIAN RANDOM FIELDS
}

\author{
KE-SEUNG LEE
}

\section{Dedicated to Professor T. Hida on the occasion of his 60 th birthday}

\section{§. Introduction}

The purpose of this paper is to investigate way of dependency of Gaussian random fields $X(D)$ indexed by a domain $D$ in $d$-dimensional Euclidean space $R^{d}$. Our main tool is variational calculus, where the boundary of a domain varies and deforms and we appeal to the white noise analysis. We therefore assume that $X(D)$ is expressed white noise integral of the form

$$
X(D)=X(D, W)=\int_{D} F(D, u) W(u) d u,
$$

where $W$ is the $R^{d}$-parameter white noise and the kernel $F(D, u)$ is a square integrable function over $R^{d}$, and where $D$ is a bounded domain with smooth boundary.

Our first interest is in the canonical property of the representation $(0,1)$, which claims that the white noise $W$ can be obtained from $X(D)$ by certain linear operations. In case this is realized, then the $W$ plays a role of the innovation for a Gaussian process with one-dimensional parameter.

Profound and, in fact, interesting results have been obtained in line with the canonical representation theory for Gaussian processes. Among others two approaches will be presented in this paper as are mentioned below:

1) The parameter $D$ is taken to be a ball

$$
B_{a}=\left\{u \in R^{d} ;|u|^{2} \leq(u, a)\right\}, \quad a \in R^{d},
$$

and $F(D, u)$ is assumed to be of the form $F(a, u) \equiv F\left(B_{a}, u\right)$ which vanishes on the boundary of $B_{a}$. Set $X\left(B_{a}\right)=X(a), a=\left(a_{1}, a_{2}, \cdots, a_{a}\right) \in R^{d}$. If the partial derivatives of $X(a)$

Received October 27, 1989. 


$$
\frac{\partial^{N}}{\partial a_{i_{1}} \cdots \partial a_{i_{N}}} X(a),
$$

exist, then they give us, as is prescribed in Section 2, the white noise $W$ (see Theorem 2.3). There the Hole theorem plays an important role. We shall see further that a property like multiple Markov property is proved for fields with kernel functions of particular type.

2) The second case is concerned with variational calculus. To fix the idea, we shall restrict our attention to the two dimensional parameter case (in Section 4 and Section 5). The random fields to be discussed are expressed in the form

$$
X\left(\left[C_{a}\right]\right)=\int_{\left[C_{a}\right]} F(u) W(u) d u,
$$

where $\left[C_{a}\right]=B_{a}$ is a disk with boundary circle $C_{a}$ with diameter $\overline{o a}$. The circle is deformed by conformal transformations and their infinitesimal deformations give us variations of $X\left(\left[C_{a}\right]\right)$. As a result, we are given a Gaussian random field of the form

$$
Y\left(C_{a}\right)=\int_{C_{a}} F(u(s)) W(u(s)) d s .
$$

Again, by applying certain conformal transformations to the parameter, the white noise $W$ is formed out of $Y\left(C_{a}\right)$. The well known theory of irreducible unitary representation of linear groups provides an essential tool for our purpose.

It is our hope that our discussion would be generalized to the case where the parameter space is more higher dimensional.

\section{§ 1. Background}

This section is provided for a brief review of some known facts on white noise analysis.

We start with a Gel'fand triple:

$$
E \subset L^{2}\left(R^{d}\right) \subset E^{*}
$$

where $E$ is a nuclear space and $E^{*}$ is the dual space of $E$. For a given characteristic functional

$$
C(\xi)=\exp \left[-\frac{1}{2}\|\xi\|^{2}\right], \quad \xi \in E,\|\cdot\| \text { the } L^{2}\left(R^{d}\right) \text {-norm, }
$$


a probability measure $\mu$ is introduced in the space $E^{*}$ in such a way that

$$
C(\xi)=\int_{E^{*}} \exp [i\langle x, \xi\rangle] d \mu(x) .
$$

The measure $\mu$ is called a white noise measure and $\mu$-almost all $x \in E^{*}$ are viewed as sample functions of white noise.

The Hilbert space $\left(L^{2}\right)=L^{2}\left(E^{*}, \mu\right)$ is the collection of all the functionals of a white noise with finite variance. A member of $\left(L^{2}\right)$ is often called a Brownian functional or a white noise functional.

A rotation $g$ of $E$ is a linear isomorphism of $E$ such that

$$
\|g \xi\|=\|\xi\|, \quad \text { for any } \xi \in E .
$$

The collection of all rotations of $E$, denoted by $O(E)$, forms a group. This group $O(E)$ is called the rotation group of $E$, or sometimes it is called an infinite-dimensional rotation group without specifying $E$.

For any $g \in O(E)$, we can find the adjoint $g^{*}$ of $g$ in such a way that

$$
\langle x, g \xi\rangle=\left\langle g^{*} x, \xi\right\rangle, \quad x \in E^{*}, \quad \xi \in E .
$$

The collection of all such $g^{*}$ with $g \in O(E)$ again forms a group and is denoted by $O^{*}\left(E^{*}\right)$ :

$$
O^{*}\left(E^{*}\right)=\left\{g^{*}: g \in O(E)\right\}
$$

If we define the product $g^{*} \circ \mu$ by

$$
\left(g^{*} \circ \mu\right)(B)=\mu\left(g^{*} B\right), \quad B \subset E^{*},
$$

then the equality

$$
g^{*} \circ \mu=\mu
$$

is valid for all $g^{*} \in O^{*}\left(E^{*}\right)$, i.e. the $\mu$ is $O^{*}\left(E^{*}\right)$-invariant.

Coming back to the rotation group $O(E)$, there are some important subgroups of $O(E)$ called whiskers: They are one-parameter subgroups of $O(E)$ satisfying the following conditions.

$$
\begin{aligned}
& \text { (i ) }\left(g_{t} \xi\right)(u)=\xi\left(\psi_{t}(u)\right)\left|\frac{\partial}{\partial u} \psi_{t}(u)\right|^{1 / 2}, \quad u \in R^{d}, \\
& \text { where } \frac{\partial}{\partial u} \psi_{t}=\left(\frac{\partial \psi_{t}^{i}}{\partial u_{j}}\right) \quad i, j=1, \cdots, d, \psi_{t}=\left(\psi_{t}^{1}, \cdots, \psi_{t}^{d}\right) \text {. } \\
& \text { (ii) } g_{t} \circ g_{s}=g_{t+s} \\
& \text { (iii) } g_{t} \text { is continuous in } t .
\end{aligned}
$$


We are specifically interested in the following four kinds of particular whiskers:

(1) shifts $S_{t}^{j}$ :

$$
\left(S_{t}^{j} \xi\right)(u)=\xi\left(u-t e_{j}\right), e_{j}=(0, \cdots, \stackrel{j}{1}, \cdots, 0) \in R^{d}, j=1, \cdots, d
$$

(2) isotropic dilation $\tau_{t}$ :

$$
\left(\tau_{t} \xi\right)(u)=\xi\left(e^{t} u\right) e^{d t / 2}, u \in R^{d},
$$

(3) rotation $\gamma_{i, j}^{\theta}$ :

$$
\left(\gamma_{i, j}^{\theta} \xi\right)(u)=\xi\left(r_{i, j}^{\theta} u\right)
$$

where $r_{i, j}^{\theta} \in S O(d)$ stands for the rotation of Euler angle $\theta$ in the $\left(x_{i}, x_{j}\right)$ plane, $i \neq j, i, j=1, \cdots, d$.

(4) special conformal transformations $\kappa_{t}^{j}=\mathfrak{w} S_{t}^{j} \mathfrak{w}$ :

$$
\left(\kappa_{t}^{j} \xi\right)(u)=\xi\left(\frac{u-t|u|^{2} e_{j}}{1-2 t u_{j}+t^{2}|u|^{2}}\right) \cdot\left(1-2 t u_{j}^{2}+t^{2}|u|^{2}\right)^{-d / 2}, \quad j=1, \cdots, d,
$$

where $\mathfrak{w}$ is the reflection with respect to the unit sphere.

There are good commutation relations among their infinitesimal generators of these whiskers, which form a $\{d(d+3) / 2+1\}$-dimensional real simple Lie algebra. Specially three classes of whiskers (2), (3) and (4) have an important property, which is the so-called "Conformal invariance property" of white noise. For details, see [10].

\section{§2. Canonical representation}

In this section we discuss canonical representations of Gaussian random fields in terms of white noise, where the parameter space is $R^{d}$, $d \geq 2$. We are particularly interested in the "canonical property" of a Gaussian random field given by white noise integral of the form

$$
X(a, W)=\int_{R^{d}} F(a, u) W(u) d u, \quad a \in R^{d},
$$

where $F(a, u)$ is a (locally) square integrable function of $u$ indexed by $a$.

Having been suggested by Hida's theory of representation for onedimensional case (see [4]), we shall adopt the following definition.

Definition 2.1. The expression (2.1) of a Gaussian random field $X(a, W)$ is canonical if for any $r>0$, the following equality holds:

$$
\sigma\{X(a, W): a \in S(r)\}=\sigma\left\{\int \chi_{S(r)}(u) \varphi(u) W(u) d u: \varphi \in L^{2}(S(r))\right\},
$$


where $\chi_{S(r)}$ is the indicator function of the ball $S(r)$ with radius $r$ and where $\sigma\{\}$ means the $\sigma$-field generated by the random variables in the bracket.

It seems difficult to find the canonical property of a general Gaussian random field of the form (2.1), so that we start with Gaussian random fields having special kernels. Such kernels do not come accidentally, but they do under the view of conformal invariance property of white noise, where the kernel $\left[(a, u)-|u|^{2}\right]$ plays an important role (see [10]). Hence we propose the following Gaussian random field:

$$
X(a, W)=\int \chi_{B_{a}}(u) P\left[(a, u)-|u|^{2}\right] f(u) W(u) d u,
$$

where $B_{a}$ is the ball with diameter $\overline{o a}$.

Before we come to this Gaussian random field, we cite

Theorem 2.1 (Hole Theorem [18]). Let $f$ be a continuous function with compact support. Suppose that the Radon transform $R f$ of $f$ vanishes on every hyperplane not intersecting a fixed compact convex set $X$. Then $f$ is zero outside of $K$.

We are now in a position to state

Theorem 2.2. The Gaussian random field (2.3) is canonical if $f$ is not equal to zero almost everywhere and rapidly decreasing at zero, and if $P$ satisfies the following conditions: there exists a natural number $k$ such that

$$
P \in C^{(k)} \quad \text { and } \quad P(0)=\cdots=P^{(k-2)}(0)=0, \quad P^{(k-1)}(0) \neq 0 .
$$

Proof. If (2.3) is not canonical, then there exist $r_{0}>0$ and $g \in L^{2}\left(S\left(r_{0}\right)\right)$, $g \neq 0$, such that

$$
\int \chi_{B_{a}}(u) P\left[(a, u)-|u|^{2}\right] f(u) g(u) d u \equiv 0,
$$

for every $a \in S\left(r_{0}\right)$. In order to use spherical harmonics expansion, let the product $\chi_{B_{a}}(u) P\left([a, u)-|u|^{2}\right]$ be expressed in terms of the polar coordinates:

$$
\tilde{P}\left[|a||u|\left(a^{\prime}, u^{\prime}\right)-|u|^{2}\right] \equiv \chi_{B_{a}}(u) P\left[(a, u)-|u|^{2}\right],
$$

where $a^{\prime}=a /|a|, u^{\prime}=u /|u|$. Then $\tilde{P} \in L^{2}\left[S^{d-1} \times S^{d-1}\right]$ as a function of $a^{\prime}$ and $u^{\prime}$. By the Funk-Hecke Theorem (cf. [16]), we have

$$
\tilde{P}\left[|a||u|\left(a^{\prime}, u^{\prime}\right)-|u|^{2}\right]=\sum_{(m, k) \in \Delta} p_{m}[|a|,|u|] S_{m, k}\left(a^{\prime}\right) S_{m, k}\left(u^{\prime}\right)
$$


where $\Delta=\{(m, k) ; m \geq 0,0 \leq k \leq h(m)\}$ with

$$
h(m)=\frac{2 m+d-2}{m+d-2}\left(\begin{array}{c}
m+d-2 \\
m
\end{array}\right),
$$

and where

$$
p_{m}(|a|,|u|)=k_{m} \int_{|u| /|a|}^{1} \tilde{P}\left[|a||u| t-|u|^{2}\right] C_{m}^{(d-2) / 2}(t)(1-t)^{2(d-3) / 2} d t .
$$

Here $C_{m}^{(d-2) / 2}$ is the Gegenbauer polynomial of degree $m$, and $k_{m}$ is the normalizing constant.

Similarly, for $Q\left[|u| u^{\prime}\right]=f(u) g(u)$, we have

$$
Q\left[|u| u^{\prime}\right]=\sum_{(m, k) \in \Delta} q_{m, k}(|u|) S_{m, k}\left(u^{\prime}\right),
$$

where $q_{m, k}(|u|)=k_{m}^{\prime} \int_{S^{d-1}} Q\left[|u| u^{\prime}\right) S_{m, k}\left(u^{\prime}\right) d \sigma\left(u^{\prime}\right)$.

Now, inserting (2.5) and (2.7) into (2.4), we get

$$
\sum_{(m, k)} k_{m}^{\prime \prime} S_{m, k}\left(a^{\prime}\right) \int_{0}^{|a|} p_{m}(|a|, s) q_{m, k}(s) s^{d-1} d s \equiv 0, \quad|a| \leq r_{0} .
$$

This yields the equation

$$
\int_{0}^{|a|} p_{m}(|a|, s) q_{m, k}(s) s^{d-1} d s \equiv 0, \quad 0<|a| \leq r_{0}, \quad(m, k) \in \Delta .
$$

From (2.6) and (2.9) we have

$$
\begin{array}{r}
\int_{0}^{|a|} q_{m, k}(s) s^{d-1}\left\{\int_{s /|a|}^{1} \cdot \tilde{P}\left[|a| s t-s^{2}\right] C_{m}^{(d-2) / 2}(t)\left(1-t^{2}\right)^{(d-3) / 2} d t\right\} d s \equiv 0, \\
\quad \text { for any }(m, k) \in \Delta .
\end{array}
$$

By changing the order of integration, we get

$$
\int_{0}^{1} C_{m}^{(d-1) / 2}(t)\left(1-t^{2}\right)^{(d-3) / 2}\{*\} d t \equiv 0, \quad(m, k) \in \Delta,
$$

where $\{*\}$ is a function of $t$ expressed in the following form:

$$
\int_{0}^{|a| t} q_{m, k}(s) \tilde{P}\left[|a| s t-s^{2}\right] s^{d-1} d s .
$$

By [15], the expression (2.11) must be a polynomial of $t$ with degree less than $m$. But $q_{m, k}(s)$ is a rapidly decreasing function of $s$ and therefore we come to know the expression (2.11) must be zero i.e., 


$$
\int_{0}^{x} q_{m, k}(s) \tilde{P}\left(x s-s^{2}\right) s^{d-1} d s \equiv 0, \quad 0 \leq x=|a| t \leq r_{0}, \quad(m, k) \in \Delta .
$$

This is a well known Volterra integral equation. The hypothesis on $P$ guarantees that $q_{m, k}(s) \equiv 0,0 \leq s \leq r_{0}$, for any $(m, k) \in \Delta$. (See [19]). We then conclude that $Q\left(|u| u^{\prime}\right)=f(u) g(u) \equiv 0$.

While the hypothesis on $f(u) \neq 0$ implies that $g(u)$ must be zero, which contradicts the choice of $g(u)$ is not zero. This completes the proof.

Remark. The function $P(y)$ in Theorem 2.2 can be replaced by $R(y)$ such as

$$
R(y)=y^{-\beta} \tilde{R}(y), \quad 0<\beta<1, \quad \tilde{R} \in C^{(1)}, \quad \tilde{R}(0) \neq 0 .
$$

(Also see [19]).

The simplest example of $P(y)$ in Theorem 2.2 is $P(y)=y^{N}$, that is,

$$
P\left[(a, u)-|u|^{2}\right]=\left[(a, u)-|u|^{2}\right]^{N} .
$$

Then the Gaussian random field

$$
X(a)=\int_{B_{a}}\left[(a, u)-|u|^{2}\right]^{N} f(u) W(u) d u
$$

is $N$-times differentiable for any $f$ satisfying the assumption given in Theorem 2.2. For such a random field, a partial converse holds.

Theorem 2.3. Let a Gaussian random field $X(a)$ be given by

$$
X(a, W)=\int_{B_{a}} F(a, u) f(u) W(u) d u,
$$

where $F(a, u)$ is a polynomial in the variables of a and $u$, where $f(u) \neq 0$ is a rapidly decreasing function at zero.

(1) If $X(a)$ is $N$-times differentiable, then $F(a, u)$ must be of the form

$$
F(a, u)=\left[(a, u)-|u|^{2}\right]^{N} F_{N}(a, u),
$$

where $F_{N}(a, u)$ does not vanish almost everywhere on the boundary of $B_{a}$.

(2) If $\partial^{N} /\left(\partial a_{i_{1}} \partial a_{i_{2}} \cdots \partial a_{i_{N}}\right) X(a)$ is an additive process for some $i_{1}, i_{2}$, $\cdot, i_{N}$, then the kernel $F_{N}(a, u)$ in (1) must be independent of $a$.

Proof. (1) Suppose that (2.13) is differentiable. Then, the difference

$$
\begin{aligned}
X\left(a^{\prime}\right)-X(a)= & \int_{B_{a}}\left[F\left(a^{\prime}, u\right)-F(a, u)\right] f(u) W(u) d u \\
& +\int\left(\chi_{B_{a}^{\prime}}(u)-\chi_{B_{a}}(u)\right) F\left(a^{\prime}, u\right) f(u) W(u) d u
\end{aligned}
$$


should have the variance of order $O\left(\left|a^{\prime}-a\right|^{2}\right)$. Noting that

$$
\int\left|\chi_{B_{a^{\prime}}}(u)-\chi_{B_{a}}(u)\right| d u
$$

is order $O\left(\left|a^{\prime}-a\right|\right)$, we can see that $F(a, u)$ must be zero on the boundary of $B_{a}$. Hence we can claim that $F(a, u)$ has a factor $(a, u)-|u|^{2}$ :

$$
F(a, u)=\left[(a, u)-|u|^{2}\right] F_{1}(a, u), F_{1}: \text { a polynomial. }
$$

Repeating this argument as many times as $N$, we finally come to the conclusion.

(2) For the proof, we refer to [11].

Remark. The random field defined by (2.12) may be said to be an $(N+1)$-ple Gaussian Markov field in the restricted sense, being a higher dimensional generalization of this notion introduced in the paper [4] by Hida.

\section{$\S 3$. Restriction of parameter}

We now pause to prepare some background in order to apply the linear operator to Gaussian random fields. The basic idea is the following: When we apply variational calculus for Gaussian random fields, we are given their values on the boundary of a domain in question. Namely, the parameter is restricted to a lower dimensional manifold. Further, we have to deal with the field even when the boundary is made to deformed. We therefore provide necessary background systematically in order to carry on the analysis of fields when the parameter is restricted.

There is a convenient tool, for this purpose, called the causal calculus. As in [6], [14] we introduce stochastic partial differential operator such as

$$
\partial_{t} ; \quad t \in R^{d}
$$

It actually stands for a partial differential operator $\partial /(\partial W(t))$ acting on functionals $f(W)$ of white noise. Since $f(W)$ can be thought of as $f\left(W(t), t \in R^{d}\right)$, where $W(t), t \in R^{d}$, is taken to be the system of variables of $f$, it is reasonable to introduce a partial derivative in the variable $W(t), t$ being fixed.

The adjoint operator $\partial_{t}^{*}$ for $\partial_{t}$ can be defined in a usual manner. Namely, 


$$
\left\langle\partial_{t} f(W), g(W)\right\rangle=\left\langle f(W), \partial_{t}^{*} g(W)\right\rangle \text {. }
$$

It can easily be seen (see [13], [14]) that each $\partial_{t}^{*}$ plays the role of the creation operator acting on the space of generalized white noise functionals.

We have, in particular,

$$
\int_{R^{d}} f(t) \partial_{t}^{*} 1 d t=\int_{R^{d}} f(t) W(t) d t, \quad f \in L^{2}\left(R^{d}\right) .
$$

Denote the operator $\int_{R^{d}} f(t) \partial_{t}^{*} 1 d t$ by $W(f)$.

As is discussed in the paper by Hida, Lee and Si Si [11], the integrand $f$ can be a generalized function, which is often taken from the space $H^{-(d+1) / 2}\left(R^{d}\right)$, the Sobolev space over $R^{d}$ of order $-(d+1) / 2$. For example, in the case where the generalized function is supported by a manifold $M\left(\subset R^{d}\right)$, the integral (3.3) may be viewed as a smeared Gaussian random variable by a generalized function defined on the parameter set $M$. Note that $M$ may be a lower dimensional manifold.

The above assertion can be justified by the following observation. Suppose $f$ in (3.3) is an $H^{-(d+1) / 2}\left(R^{d}\right)$-function. Then, we can provide a test functional which is a Gaussian random variable given by

$$
\int \xi(t) W(t) d t=W(\xi)
$$

where $\xi \in H^{(d+1) / 2}\left(R^{d}\right)$. The bilinear form $\langle W(f), W(\xi)\rangle$ has the value $\langle f, \xi\rangle$ as is well known. Note that $\langle f, \xi\rangle=\left\langle f, \xi^{M}\right\rangle$ holds, where $\xi^{M}$ is the restriction of $\xi$ to $M$. This means that $f$ may be viewed as a (generalized) function on $M$. Now, coming back to $W(f)$, we understand that, so far as $f$ runs through the class of generalized functions with support $M, W(f)$ can be dealt with as if it were a linear functional of the white noise on $M$ evaluated at $f$, although it can never be an ordinary white noise functional. For details, see [9].

Remark. Here one can also see an advantage of using the integral representation introduced in [5].

\section{§4. Subgroup of $\tilde{C}(2)$}

In this section we restrict our attention to the two-dimensional parameter case. The conformal group is now $C(2)$, which involves two 
shifts, the isotropic dilation, the rotation on $R^{2}$ and two special conformal transformations. Now introduce a notation $\tilde{g}$ which denotes a diffeomorphism of $\bar{R}^{2}=R^{2} \cup\{\infty\}$ such that $g \in C(2)$ can be expressed in the form

$$
(g \xi)(u)=\xi(\tilde{\mathrm{g}} u)|J(\tilde{\mathrm{g}} u)|^{1 / 2}, \quad J \text { being the Jacobian. }
$$

We set

$$
\tilde{C}(2)=\{\tilde{g} ; g \in C(2)\} .
$$

Let $S$ be the collection of all plane circles and let $S_{0}$ be the subset of $S$ which consists of circles passing through the origin. There are two interesting subgroups of $\tilde{C}(2)$ in connection with $S_{0}$ :

$$
\begin{aligned}
& \tilde{I}_{0}=\left\{\tilde{\boldsymbol{g}} \in \tilde{C}(2): \tilde{\boldsymbol{g}} S_{0}=S_{0}\right\}, \\
& \tilde{I}\left(C_{a}\right)=\left\{\tilde{\boldsymbol{g}} \in \tilde{C}(2): \tilde{\mathrm{g}} C_{a}=C_{a}\right\}, \quad C_{a} \in S_{0},
\end{aligned}
$$

where $C_{a}$ is the circle having $a$ as the antipodal point to the origin.

It is easy to see that $\tilde{I}_{0}$ is generated by $\left\{\tilde{\gamma}_{\theta}, \tilde{\tau}_{t}, \tilde{\kappa}_{t}^{1}, \tilde{\kappa}_{t}^{2}\right\}$. This group $\tilde{I}_{0}$, acting on $S_{0}$, is transitive.

The family $\left\{\tilde{I}\left(C_{a}\right) ; a \in R^{2}\right\}$ is a system of subgroup of the $\tilde{I}_{0}$ such that $\tilde{I}\left(C_{a}\right) \cap \tilde{I}\left(C_{b}\right)=\{$ identity map $\}, a \neq b$. Every member of the $\tilde{I}\left(C_{a}\right)$ comes from the suitable combination of $\left\{\tilde{\tau}_{t}, \tilde{\kappa}_{t}^{1}, \tilde{\kappa}_{t}^{2}\right\}$. To find an expression of the member of $\tilde{I}\left(C_{a}\right), a=\left(a_{1}, a_{2}\right)$, set

$$
g_{\tau}^{i}=\tau_{\log \left(1-a_{i} t\right)} \kappa_{t}^{i}, \quad i=1,2,
$$

where $\tau$ is a function of $t$. Noting the commutation relation

$$
\kappa_{t}^{i} \tau_{s}=\tau_{s} \kappa_{t \cdot \exp [s]}^{i}
$$

we take $\tau$ to be $\left(\log \left(1-a_{i} t\right)\right) / a_{i}$ so that $g_{\tau}^{i}$ becomes a one-parameter group, $i=1,2$.

The infinitesimal generator $\alpha_{i}$ of $g_{\{t\}}^{i}$ turns out to be

$$
\alpha_{i}=\left.\frac{d}{d \tau} g_{\tau}^{i}\right|_{\tau=0}=a_{i} \tau-\kappa^{i}, \quad i=1,2,
$$

where $\tau$ and $\kappa^{i}$ are infinitesimal generators of $\tau_{t}$ and $\kappa_{t}^{i}$, respectively.

The commutation relation between $\alpha_{i}, i=1,2$, is

$$
\begin{aligned}
{\left[\alpha_{1}, \alpha_{2}\right] } & =a_{2} \kappa^{1}-a_{1} \kappa^{2} \\
& =a_{1} \alpha_{2}-a_{2} \alpha_{1} .
\end{aligned}
$$

If we set $\beta=a_{1} \alpha_{2}-a_{2} \alpha_{1}$, then we have 


$$
\left[\alpha_{i}, \beta\right]=a_{i} \beta, \quad i=1,2 .
$$

Summing up these results, we obtain

Proposition 4.1. Let $C_{a}, a=\left(a_{1}, a_{2}\right)$, be given. Then the infinitesimal generators $\left\{\alpha_{i}\right\}$ of $\left\{g_{r}^{i}\right\}$ span a two-dimensional Lie algebra:

$$
\begin{aligned}
V\left[\alpha_{1}, \alpha_{2}\right] & =V\left[\alpha_{1}, \beta\right], & & a_{1} \neq 0, \\
& =V\left[\alpha_{2}, \beta\right], & & a_{2} \neq 0,
\end{aligned}
$$

where $V$ is the vector space with the product in (4.3).

\section{§5. Variational calculus based on conformal group}

We are now in a position to investigate the Gaussian random field $X(a), a \in R^{2}$, by exploiting the one parameter groups that have been prepared in $\S 4$.

Let $X(a)$ be given by

$$
X(a)=\int_{B_{a}} F(u) W(u) d u,
$$

where $F(u)$ is a locally square integrable function.

First, we apply $\kappa_{t}^{1}$ to $X(a)$ to obtain

$$
\begin{aligned}
& \left(\delta_{\kappa_{1}} X\right)(a)=\left.\frac{d}{d t}\left(\kappa_{t}^{1} X\right)(a)\right|_{t=0} \\
& \quad=\int_{C_{a}} F(u) \frac{a_{1}}{|a|}|u|^{2} W(u) d \tilde{u}+\int_{B_{a}}\left(\kappa^{1} F\right)(u) W(u) d u,
\end{aligned}
$$

where the $d \tilde{u}$, in the first integral, denotes the line elements $d s$ and where $W(u)$, in the first integral, must be understood as the restriction of $W$ to the boundary $C_{a}$ as was discussed in Section 3,

Similarly, applying $\kappa_{t}^{2}$ and $\tau_{t}$ to (5.1), we get

$$
\begin{aligned}
\left(\delta_{\kappa^{2}} X\right)(a) & =\left.\frac{d}{d t}\left(\kappa_{t}^{2} X\right)(a)\right|_{t=0} \\
& =\int_{C_{a}} F(u) \frac{a_{2}}{|a|}|u|^{2} W(u) d \tilde{u}+\int_{B_{a}}\left(\kappa^{2} F\right)(u) W(u) d u,
\end{aligned}
$$

and

$$
\begin{aligned}
\left(\delta_{\tau} X\right)(a) & =\left.\frac{d}{d t}\left(\tau_{t} X\right)(a)\right|_{t=0} \\
& =\int_{C_{a}} F(u) \frac{|u|^{2}}{|a|} W(u) d \tilde{u}+\int_{B_{a}}(\tau F)(u) W(u) d u,
\end{aligned}
$$


respectively.

In the equations of (5.2), (5.3) and (5.4), the two integral can be discriminated by evaluating their variances; more precisely, the first term is of infinite order from our understanding that the kernel function is a generalized function, while the second term has order $O(\sqrt{\delta a})$, where $\delta a$ denotes the differential of $a \in R^{2}$. We can therefore choose the first integral terms which are dominant.

Now the first integral can be rewritten as the following expression:

$$
Y(a)=Y\left(C_{a}\right)=\int_{C_{a}} G(a, u(s)) W(u(s)) d s,
$$

where we should note that $G(a, u)(1 /|u|)^{2}$ is a square integrable function. This looks like an ordinary Gaussian random field. We can therefore apply to the $Y(a)$ the transformations that come from the prepared one parameter groups $\left\{g_{\tau}^{i}\right\}$ and we obtain the following expressions:

For $g_{\tau}^{1}$, we have

$$
\begin{aligned}
\left(\delta_{g^{1}} Y\right)\left(c_{a}\right) & =\left.\frac{d}{d \tau}\left(g_{\tau}^{1} Y\right)\left(C_{a}\right)\right|_{\tau=0} \\
& =\int_{C_{a}}\left(\alpha_{1} G\right)(u) W(u)\left(r^{2}+r^{\prime 2}\right)^{1 / 2} d u(s),
\end{aligned}
$$

where $r=a_{1} \cos \theta+a_{2} \sin \theta, r^{\prime}=-a_{1} \sin \theta+a_{2} \cos \theta$, and where $\theta=s /|a|$ $-\tan ^{-1}\left(a_{1} / a_{2}\right)$.

Similarly, for $g_{r}^{2}$, we have

$$
\begin{aligned}
\left(\delta_{g^{2}} Y\right)\left(C_{a}\right) & =\left.\frac{d}{d \tau}\left(g_{\tau}^{2} Y\right)\left(C_{a}\right)\right|_{\tau=0} \\
& =\int_{C_{a}}\left(\alpha_{2} G\right)(u) W(u)\left(r^{2}+r^{\prime 2}\right)^{1 / 2} d u(s) .
\end{aligned}
$$

Without loss of generality, we may set $a=\left(0, a_{2}\right)$ to avoid unessential complexity. With this assumption we observe the following transformations. By the reflection $\mathfrak{w}$, the elements $\boldsymbol{u}=(u, v) \in C_{a}, \quad \tilde{\boldsymbol{g}}_{\tau}^{1} \boldsymbol{u} \in C_{a}$, and $\tilde{g}_{\tau}^{2} u \in C_{a}$ are transformed to

$$
\frac{1}{a_{2}}\left(\frac{u}{v}, 1\right), \frac{1}{a_{2}}\left(\frac{u}{v}+a_{2} t, 1\right) \text { and } \frac{1}{a_{2}}\left(e^{-a_{2} \tau} \frac{u}{v}, 1\right),
$$

respectively.

Then $\tilde{g}_{\{t\}}^{1}$ plays as translation operator, while $\tilde{\mathbf{g}}_{\{t\}}^{2}$ does as the dilation operator. If we add the operator $\widetilde{\text { ef }_{a}}$, which is the reflection with respect 
to the line passing through the origin and its antipodal point $a$, then the group $\mathfrak{c}_{a}$ generated by the subgroups $\left\{\tilde{g}_{\{t\}}^{1}, \tilde{g}_{\{t\}}^{2}, \widetilde{\text { ref }} a\right\}$ admits an irreducible unitary representation on the Hilbert space spanned by the $W(f), f$ being a function on $C_{a}$. Thus, if we invoke the result by Gel'fand and Naimark [3], we can pick out the white noise $W(u), u \in C_{a}$, and eventually $W(u)$, $u \in R^{2}$, out of the original random field $X(a), a \in R^{2}$. From this result, the $\{W(u)\}$ may be thought of as a generalization of "innovation" using the action of the group $\tilde{C}(2)$.

Now we summarize the above results as a theorem:

Theorem 5.1. Let $X(a)$ be the Gaussian random field given by the formula (5.1). Then,

(1) we can form Gaussian random fields $Y(a)$ as in (5.5) by applying $\tilde{I}\left(C_{a}\right)$ to $X(a)$ in $(5.1)$ :

(2) we can recover the original white noise $W(u)$ by applying the transformation group $\mathfrak{\aleph}_{a}$ to $Y(a)$.

Concluding Remark

Before closing this article, we state some remarks.

In Section 4 and Section 5, we have used variational calculus for only two-dimensional case and the domain of integration are chosen from much restricted class i.e. $C_{a}, a \in R^{2}$.

It seems that there are at least two directions for further development in line with our approach:

(1) generalization to higher dimensional case, and

(2) generalization of the choice for domains of integraticn

For the first direction, an innovation process may be obt: $d$, using $C(d), d \geq 3$, by a similar but somewhat more complicated meinod with the same idea.

For the second direction, we note that some results for a more general class of curves are reported in Si Si [17] and in Section 5 of Hida and Si Si [12].

\section{Acknowledgements}

The author is glad to express his thanks to Professor T. Hida, and Professor A. Noda as well as other probabilists at Nagoya area. Professor Noda gave the author valuable suggestions and suggested improvements for Theorem 2.2. Especially Professor Hida has encouraged the author; without his help, it seems impossible to the author to complete this paper. 


\section{REFERENCES}

[1] P. Lévy, Processus stochastiques et mouvment brownein, Gauthier-Villars, Paris, 1948.

[2] - A special problem of Brownian motion and a general theory of Gaussian random function, Proc. 3rd Berkeley Symp., Vol. II (1956), 133-175.

[ 3 ] I. M. Gel'fand and M. A. Naimark, Unitary representation of the group of linear transformations of the straight line, Comptes Rendus (Doklady) de l'Acade'mie des Sciences de l'URSS (1947), Vol. 55, No.7, 567-570.

[4] T. Hida, Canonical representation of Gaussian process and their applications, Memo. Coll. Sci., Univ. of Kyoto, 33 (1960), 109-351.

[5] —-, Brownian Motion (in Japanese), Iwanami Pub. Co., Tokyo (1975) ; English Trans. Springer-Verlag, New York, Heidelberg, Berlin (1980) ; Russian Trans., Nauka, Moscow, 1987.

[6] T. Hida, Analysis of Brownian functionals, Carleton Mathematical Lecture Notes, no. 13 (1975), and 2nd ed. (1978).

[7] — A note on generalized Gaussian random fields, Jour. Multivariate Analysis, 27 (1988), 255-260.

[ 8 ] - White noise analysis and Gaussian random field, Proc. 24th Karpacz Winter School of Theoretical Physics, 1988, Stochastic Methods in Mathematics and Physics. World Scientific, 277-289.

[9] — - White noise and Gaussian random fields, Proc. Singapore Probability Conference, June 1989, ed. Louis H. Y. Chen et al., to appear.

[10] T. Hida, K.-S. Lee and S.-S. Lee, Conformal invariance of white noise, Nagoya Math. J., 98 (1985), 87-98.

[11] T. Hida, K.-S. Lee and Si Si, Multidimensional parameter white noise and Gaussian random fields, Balakrishnan Volume (1987), 177-183.

[12] T. Hida, Si Si, Variational calculus for Gaussian random fields, Proc. IFIP, Warsaw, 1988; Stochastic Systems and Optimization, Lecture Notes in Control and Information Sci. no. 136, ed. J. Zabczyk (1989), 86-97.

[13] T. Hida, H.-H. Kuo, J. Pottoff and L. Streit, White noise: an infinite-dimensional calculus, monograph in preparation.

[14] I. Kubo and S. Takenaka, Calculus on Gaussian white noise, I-IV, Proc. Japan Academy A, Math. Sci., 56 (1980) 376-380, 411-416, 57 (1981) 433-437, 58 (1982) 186-189.

[15] A. Noda, Generalized Radon transform and Lévy's Brownian motion I, II, Nagoya Math. J., 105 (1987), 71-87 and 89-107.

[16] R. T. Seeley, Spherical harmonics, Amer. Math. Monthly, 73 (1966), 115-121.

[17] Si Si, A note on Lévy's Brownian Motion, I, II, Nagoya Math. J., 108 (1987), 121-130 and 114 (1989), 165-172.

[18] R. S. Strichartz, Radon inversion-variations on a theme, Amer. Math. Monthly, 89 (1982), 377-384 and 420-423.

[19] K. Yosida, Lectures on differential and integral equations, Interscience Pub., 1960.

College of Liberal Arts and Sciences

Department of Mathematics

Korea University

Chochiwan, Korea 